УДК 621.315 .592

\title{
Высокоомный антимонид галлия, полученный методом газофазной эпитаксии из металлорганических соединений
}

\author{
(C) Р.В. Левин, А.С. Власов, А.Н. Смирнов, Б.В. Пушный \\ Физико-технический институт им. А.Ф. Иофрфе Российской академии наук, \\ 194021 Санкт-Петербург, Россия \\ E-mail: Lev@vpegroup.ioffe.ru
}

Поступила в Редакцию 10 июля 2019 г.

В окончательной редакции 15 июля 2019 г.

Принята к публикации 15 июля 2019 г.

\begin{abstract}
Представлены результаты исследований преднамеренно не легированных эпитаксиальных слоев $p$-GaSb, выращенных методом газофазной эпитаксии из металлорганических соединений при соотношении $\mathrm{TMSb} / \mathrm{TEGa}$ от 1 до 50. При соотношении TMSb/TEGa $=50$ были изготовлены эпитаксиальные слои $\mathrm{GaSb}$ с удельным электрическим сопротивлением 400 Ом - см. Показано, что кристаллическое качество таких слоев, которое оценивалось несколькими методами, оставалось соизмеримым с качеством подложек $(n-\mathrm{GaSb})$ используемых для роста преднамеренно не легированных слоев $\mathrm{GaSb}$.
\end{abstract}

Ключевые слова: газофазная эпитаксия, подложка, высокоомный антимонид галлия, кристаллическое качество.

DOI: 10.21883/FTP.2019.12.48609.9208

\section{1. Введение}

Серьезным недостатком узкозонных полупроводниковых соединений $\mathrm{A}^{\mathrm{III}} \mathrm{B}^{\mathrm{V}}$ при изготовлении интегральных схем является отсутствие собственной полуизолирующей подложки.

Использование $\mathrm{GaSb}$ в качестве подложек дает возможность изготавливать согласованные по постоянной кристаллической решетки слои твердых растворов (Al)GaInAsSb в широком диапазоне составов с шириной запрещенной зоны в диапазоне $E_{g} \sim 0.3-1.6$ эВ (0.8-4.1 мкм), что привлекательно для оптоэлектроники.

Преднамеренно не легированный GaSb в независимости от технологии и условий изготовления получается $p$-типа проводимости [1]. При этом концентрация акцепторов обычно составляет $\sim 10^{17} \mathrm{~cm}^{-3}$ и в основном связана с вакансиями сурьмы $\left(V_{\mathrm{Sb}}\right)$ и замещением атомов $\mathrm{Sb}$ атомами $\mathrm{Ga}\left(\mathrm{Ga}_{\mathrm{Sb}}\right)$ в кристаллической решетке. Как показано в работе [2], выращивание методом жидкофазной эпитаксии (ЖФЭ) слоев $\mathrm{GaSb}$ из раствора-расплава в присутствии нейтрального „растворителя“ позволяет увеличить количество атомов сурьмы и снизить концентрацию таких вакансий. В результате чего было достигнуто удельное сопротивление материала слоев 400 Ом · см при $T=77 \mathrm{~K}$.

Существенным ограничением технологии ЖФЭ является низкая производительность и малые площади структур, что препятствует его использованию в производстве. При газофазной эпитаксии из металлорганических соединений (ГФЭМОС) достаточно легко получить превышение содержания атомов сурьмы по отношению к содержанию атомов галлия в газовой фазе в 20-50 раз. Кроме того, метод ГФЭМОС на данный момент является наиболее производительным и позволяет получать слои $\mathrm{GaSb}$ большей площади и однородности, что важно для промышленного производства полупроводниковых приборов.

Цель данной работы - исследование возможности изготовления эпитаксиальных слоев $\mathrm{GaSb}$ с большим удельным электрическим сопротивлением методом ГФЭМОС.

\section{2. Эксперимент и обсуждение результатов}

Bce слои $\mathrm{GaSb}$ выращивали методом ГФЭМОС на подложках, ориентированных в плоскости (100) $n$-GaSb:Te, и полуизолирующих подложках (100) $i$-GaAs необходимых для проведения холловских измерений, так как для $\mathrm{GaSb}$ отсутствуют собственные полуизолирующие подложки. Температура роста была $600^{\circ} \mathrm{C}$, а давление в реакторе - 100 мбар при суммарном потоке водорода через реактор, равном 5.5 л/мин. В качестве источников использовали триметилсурьму (TMSb) и триэтилгаллия (TEGa). Соотношение молярных потоков TMSb/TEGa было в диапазоне 1-50. Поток TEGa был постоянным (28.6 мкмоль/мин), а соотношение молярных потоков TMSb/TEGa менялось за счет изменения потока TMSb. Рост осуществлялся в течение 1.5 ч. Толщина выращенных слоев составляла 1.2-1.8 мкм.

На рис. 1 приведена зависимость удельного электрического сопротивления $\mathrm{GaSb}$ от соотношения молярных потоков TMSb/TEGa. Видно, что с увеличением соотношения TMSb/TEGa наблюдается увеличение удельного электрического сопротивления в эпитаксиальных слоях. Удельное электрическое сопротивление > $400 \mathrm{OM} \cdot \mathrm{cm}$ было определено для слоев, выращенных при соотношении потоков элементов TMSb/TEGa равном 50. 


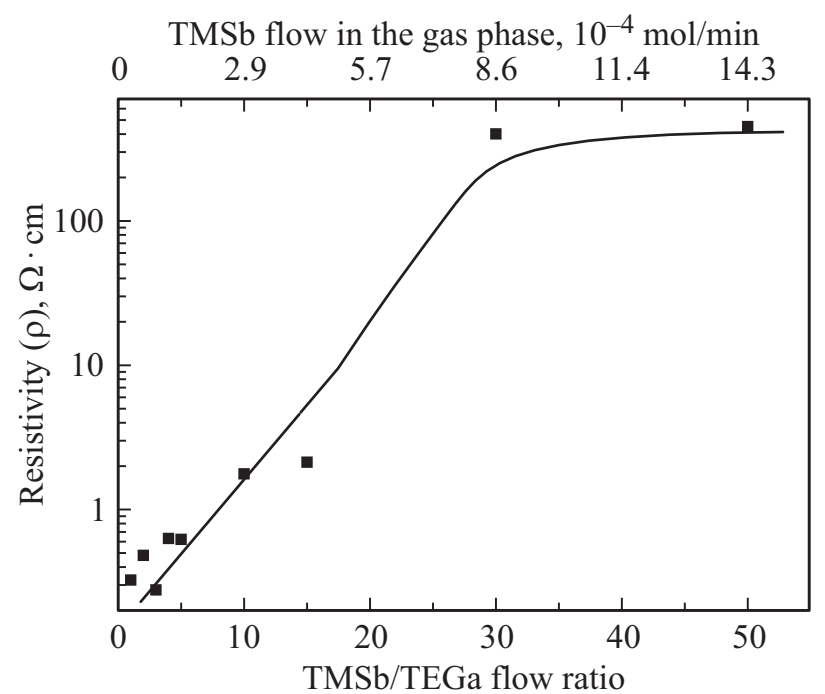

Рис. 1. Зависимость удельного электрического сопротивления $p$-GaSb от соотношения молярных потоков TMSb/TEGa.

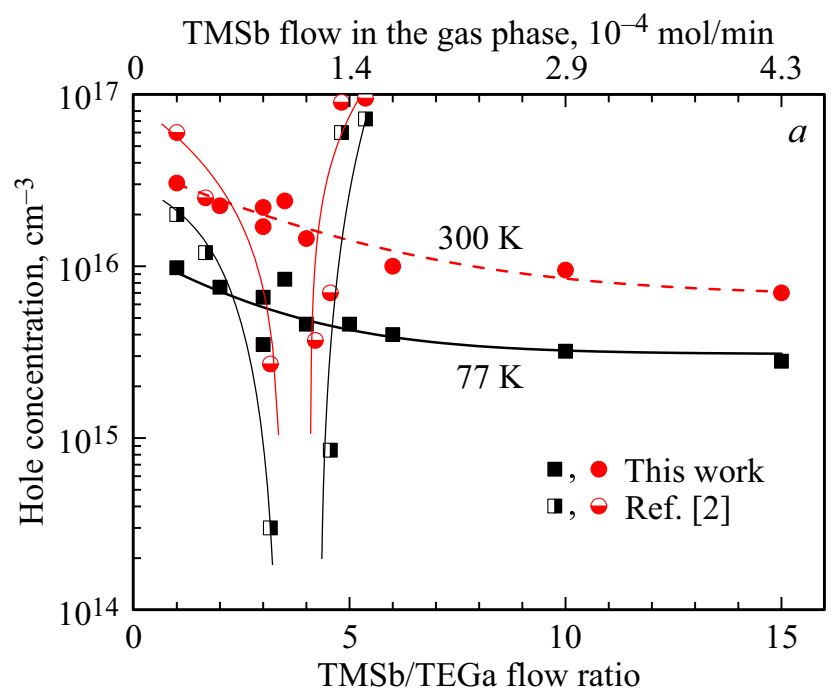

TMSb flow in the gas phase, $10^{-4} \mathrm{~mol} / \mathrm{min}$

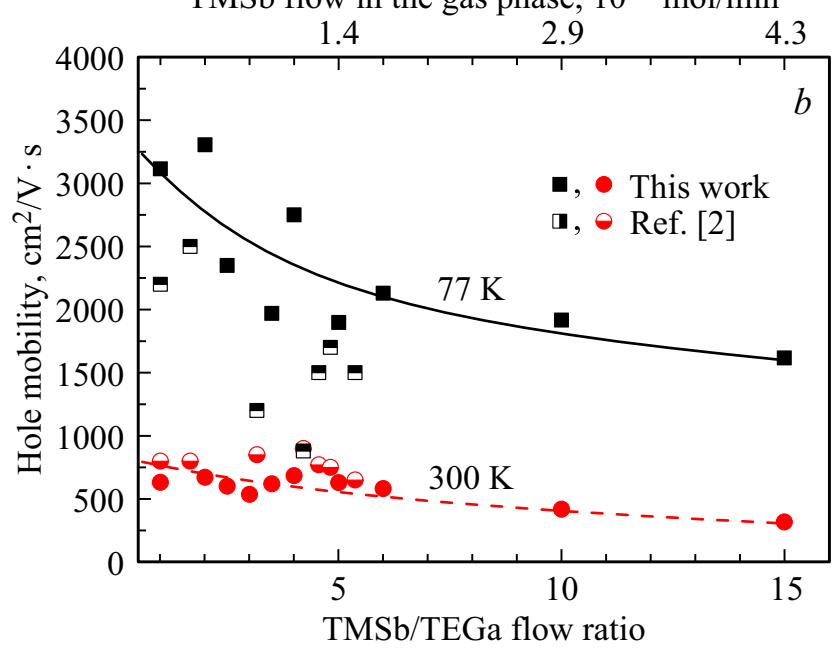

Рис. 2. Зависимость концентрации (a) и подвижности $(b)$ дырок в $p$-GaSb от соотношения молярных потоков TMSb/TEGa для слоев, выращенных на подложках полуизолирующего GaAs. На рисунке также представлены данные из работы [2].
Данные по концентрации и подвижности получены с помощью 6-зондового метода Холла при температуре 77 и $300 \mathrm{~K}$ для слоев GaSb, выращенных на подложках полуизолирующего GaAs. Было установлено (см. рис. 2), что эпитаксиальные слои антимонида галлия в диапазоне соотношений TMSb/TEGa от 1 до 15 имели $p$-тип проводимости. При соотношении TMSb/TEGa более 20 такие измерения были невозможны.

Как видно из рис. 2, $a$, концентрация дырок монотонно убывает при увеличении концентрации сурьмы в газовой фазе. Это может указывать на снижение концентрации дефектов, связанных со стехиометрией $V_{\mathrm{Sb}} \mathrm{Ga}_{\mathrm{Sb}}$ [2]. При этом подвижность (рис. 2,b) дырок также снижается, что, по-видимому, свидетельствует об увеличении электрически неактивных центров, на которых происходит рассеивание носителей заряда.

Для сравнения на рис. 2 добавлены экспериментальные значения концентрации и подвижности, полученные в работе [2], при этом значения концентрации сурьмы в жидкой фазе пересчитаны на значение соотношения $\mathrm{Sb} / \mathrm{Ga}$ в диапазоне 1-7. Из данных, представленных на рис. $2, b$, видно, что значения подвижностей, полученные в работе [2], соизмеримы со значениями, полученными в данной работе, тогда как изменение концентрации имеет особенности (провал) при соотношении $\mathrm{Sb} / \mathrm{Ga}$ в диапазоне 3-4.

Был выполнен ряд исследований структурных и люминесцентных свойств выращенных высокоомных слоев $\mathrm{GaSb}$ для оценки кристаллического совершенства материала.

1. Кристаллическое совершенство слоев оценивалось при помощи измерений, на установке рентгеновской дифракции ДРОН-2 (монохроматор $\mathrm{Ge}(001))$, излучение $\mathrm{Cu} K_{\alpha}(0.154$ нм $)$, при размере щелей 1 мм. На рис. $3, a$ приведены для сравнения характерные рентгенодифракционные кривые качания вблизи рефлекса (004) $\mathrm{GaSb}$ эпитаксиальных слоев, выращенных на подложках $\mathrm{GaSb}$ при соотношении $\mathrm{TMSb} / \mathrm{TEGa}=2,10,50$, и для используемой подложки $n-\mathrm{GaSb}:$ Те. Полная ширина на половине высоты (FWHM) рентгенодифракционной кривой качания подложки без эпитаксиального слоя составляла 44", тогда как FWHM кривых качания для приведенных на рис. 3, $a$ слоев $p$-GaSb, выращенных на таких подложках, были в диапазоне 19-30", что указывает на улучшение кристаллической структуры эпитаксиального слоя по сравнению с подложкой. На вставке рис. 3, a приведена зависимость FWHМ кривых качания от соотношения молярных потоков TMSb/TEGa в диапазоне $1-50$, которая показывает рост от наименьшего значения FWHM 17-20" для слоев, выращенных при $\mathrm{TMSb} / \mathrm{TEGa} \leq 10$ до значений $\mathrm{FWHM}=35^{\prime \prime}$ при $\mathrm{TMSb} / \mathrm{TEGa}=30$. На рис. $3, b$ приведено соотношение величины удельного сопротивления в исследуемых слоях $p$-GaSb и FWHM. Из приведенной зависимости видно, что с увеличением FWHМ увеличивается удельное сопротивления слоев. Таким образом, опираясь на данные рентгеноструктурного анализа, можно сделать 

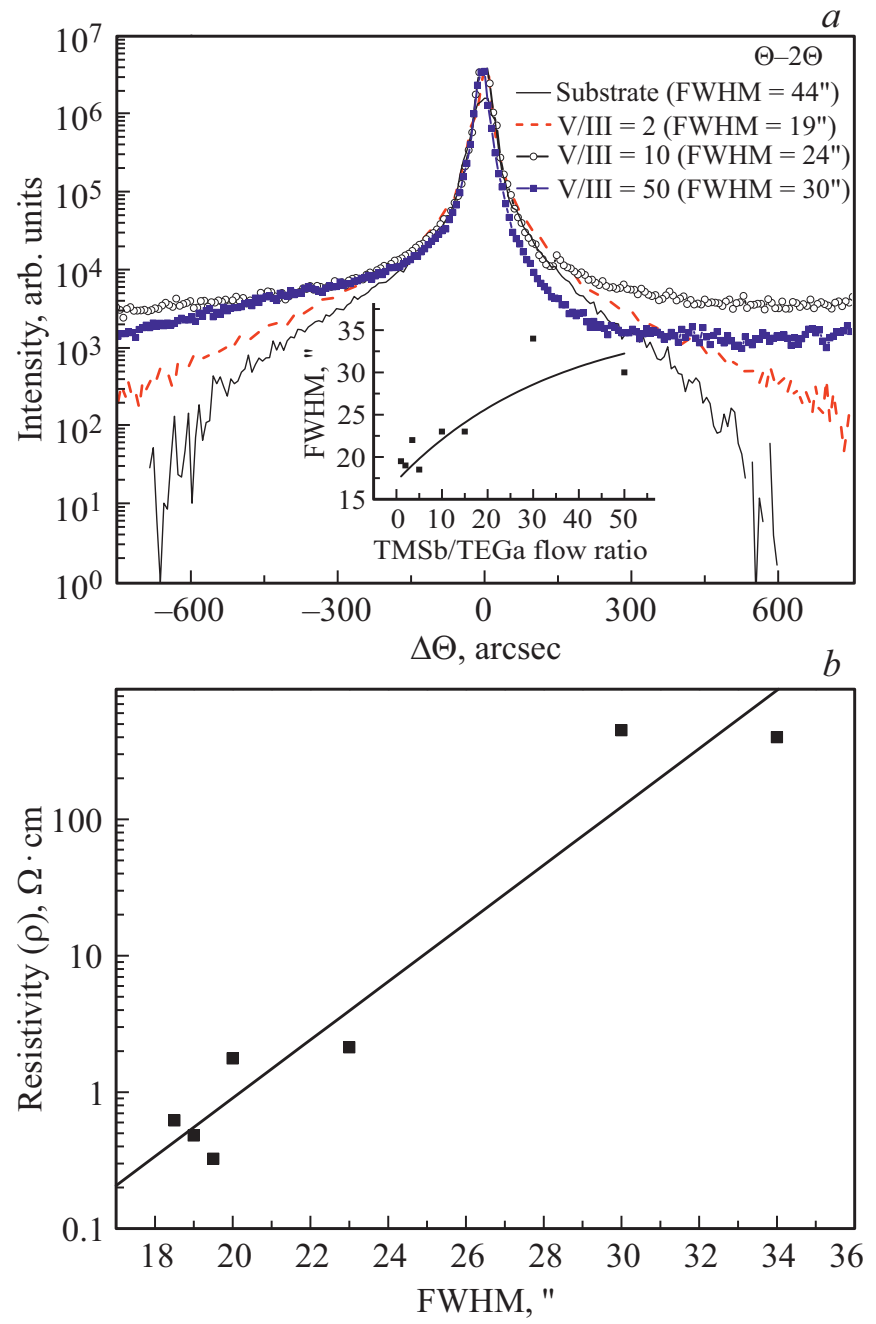

Рис. 3. $a-$ типичные рентгенодифракционные кривые качания для используемой подложки и слоев $p$-GaSb, выращенных при $\mathrm{TMSb} / \mathrm{TEGa}=2,10$ и 50. На вставке приведена зависимость FWHM рентгенодифракционной кривой качания от соотношения молярных потоков TMSb/TEGa в диапазоне 1-50. $b-$ соотношение величин удельного сопротивления и FWHM рентгенодифракционных кривых качания в исследуемых слоях $p-\mathrm{GaSb}$.

вывод, что при увеличении сопротивления (см. рис. $3, b$ ) количество структурных дефектов в слоях незначительно возрастает и остается соизмеримым в сравнении с подложкой, при этом удельное электрическое сопротивление увеличилось более чем на 3 порядка величины.

2. Исследование фотолюминесцентных характеристик слоев было выполнено при температуре жидкого азота $(77 \mathrm{~K})$. Для возбуждения использовался CW Nd:YAG-лазер (532 нм). Плотность мощности составляла $\sim 10-300 \mathrm{Bт} / \mathrm{cm}^{2}$. Излучение детектировалось с помощью $\mathrm{PbS}$ фоторезистора.

На вставке рис. 4 для сравнения приведены характерные спектры фотолюминесценции (ФЛ) исходной подложки $\mathrm{GaSb}$ и слоев $p$ - GaSb, выращенных при разных соотношениях молярных потоков $\mathrm{TMSb} / \mathrm{TEGa}=2,10$ и 50. В спектре можно выделить полосу межзонной рекомбинации с энергией $\sim 800 \mathrm{MB}$, полосу с энергией 0.775 эB, связанную с $V_{\mathrm{Ga}} \mathrm{Ga}_{\mathrm{Sb}}$ (которая наблюдается для слоев, выращенных при TMSb/TEGa $\leq 2$, а при дальнейшем увеличении соотношения TMSb/TEGa эта полоса подавляется, см. вставку на рис. 4,a), и так называемую $T$-полосу, соответствующую донорно-акцепторным переходам с участием однозарядного акцепторного комплекса, образующегося в результате взаимодействия атомов Те (так как использовались подложки $\mathrm{GaSb}$ с концентрацией Те $\left.\sim(1-5) \cdot 10^{17} \mathrm{~cm}^{-3}\right)$ и собственных дефектов, являющихся двухзарядными акцепторами $[3,4]$. Ввиду того что коэффициент диффузии атомов Те считается низким $\left(D=(1-3) \cdot 10^{-5} \mathrm{~cm}^{2} / \mathrm{c}[5]\right)$, мы полагаем, что данный сигнал связан с подложкой, на которой выращивался слой. При сравнении спектров ФЛ слоев $p$-GaSb, выращенных при разных TMSb/TEGa, можно отметить, что с увеличением TMSb/TEGa от 10 до 50 интенсивность полосы межзонной рекомбинации снижается, тогда как интенсивность $T$-полосы увеличивается, при этом наблюдается ее сдвиг в сторону высоких энергий, что связано с увеличением энергии переходов.

На рис. 4 приведены данные по FWHM пика межзонной рекомбинации для образцов, выращенных при различных соотношениях молярных потоков TMSb/TEGa. Из рисунка видно, что FWHM пика ФЛ хорошо коррелирует с условиями роста: наименьшее значение наблюдается при соотношении потоков V/III $=2$, тогда как при меньших значениях (рост в избытке $\mathrm{Ga}$ ) и больших значениях (рост в избытке $\mathrm{Sb}$ ) наблюдается последовательное увеличение ширины полосы излучения.

Таким образом, из анализа спектров ФЛ можно сделать вывод об увеличении концентрации собственных

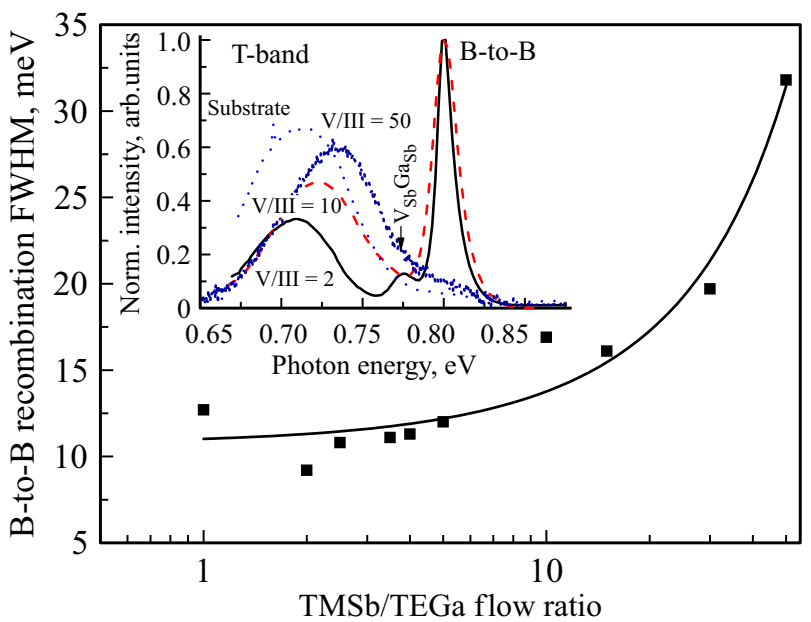

Рис. 4. Зависимость FWHM пика межзонной рекомбинации для образцов, выращенных при различных соотношениях молярных потоков TMSb/TEGa. На вставке приведены характерные спектры ФЛ эпитаксиальных слоев $\mathrm{GaSb}$, полученных при $\mathrm{TMSb} / \mathrm{TEGa}=2$ (сплошная), 10 (пунктирная), 50 (штрихпунктирная), и используемой подложки $\mathrm{GaSb}$ (точечная), измеренные при $T=77 \mathrm{~K}$. 


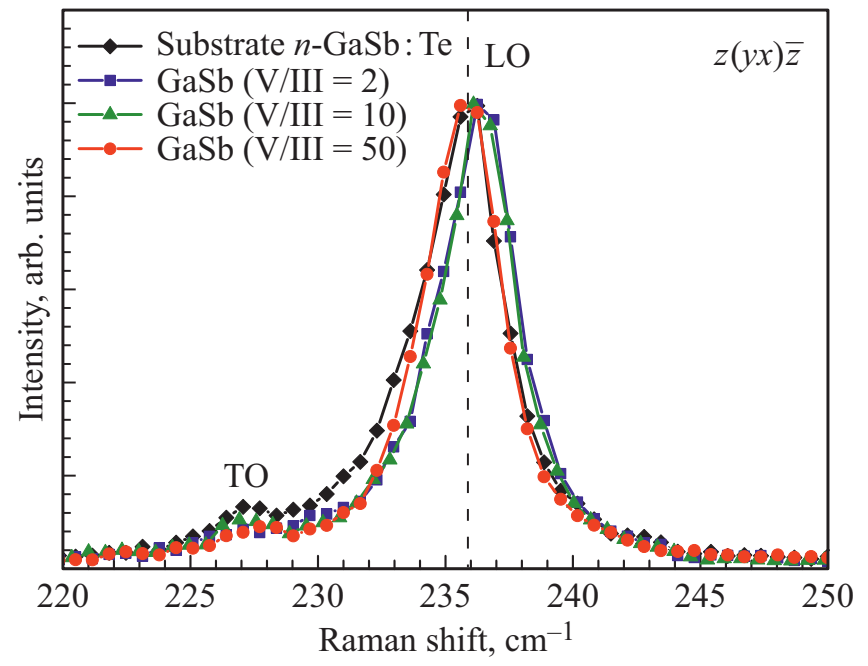

Рис. 5. Спектры КРС подложки $n$-GaSb:Te $\left(n \sim 3 \cdot 10^{17} \mathrm{~cm}^{-3}\right)$ и преднамеренно не легированных слоев $\mathrm{GaSb}$, полученных при соотношении молярных потоков $\mathrm{TMSb} / \mathrm{TEGa}=2,10$ и 50 $(T=300 \mathrm{~K})$. Вертикальной пунктирной линией показано частотное положение LO-фонона для объемного недеформированного $\mathrm{GaSb}$.

дефектов, наличие которых приводит к сдвигу уровня Ферми [6] в глубь запрещенной зоны. Согласно данным работы [7], это могут быть дефекты замещения $\mathrm{Sb}_{\mathrm{Ga}}$, которые имеют тенденцию трансформироваться в электрически нейтральный комплекс $V_{\mathrm{Ga}} \mathrm{Sb}_{i}$.

3. На исходной подложке $n$-GaSb:Te и образцах $p$-GaSb, выращенных при разных соотношениях молярных потоков (TMSb/TEGa = 2, 10, 50), были выполнены исследования спектров комбинационного рассеяния света (КРС) при комнатной температуре на спектрометрической установке Horiba Jobin-Yvon T64000, оснащенной конфокальным микроскопом. Для возбуждения спектров рассеяния использовался $\mathrm{Ar}^{+}$-лазер (длина волны $\lambda=514$ нм). Плотность лазерного излучения на образце была 2.5-50кВт/см². Умеренный уровень плотности возбуждения исключал локальный нагрев образца и позволял избежать эффекта, при котором концентрация фотовозбужденных носителей достигает существенных значений, способных оказать влияние на спектры КРС. Измерения спектров проводились в геометрии обратного рассеяния $z(x y) \bar{z}$, где направление $z$ совпадает с направлением роста пленок $\mathrm{GaSb}$, а оси $x$ и $y$ ортогональны и расположены в плоскости образца.

На рис. 5 представлены спектры КРС исследованных слоев $p$-GaSb и исходной подложки. В спектрах наблюдаются основная линия $\left(\omega \sim 236 \mathrm{~cm}^{-1}\right)$, связанная с рассеянием света на продольном (LO) фононе, и слабая особенность на частоте $\omega \sim 227 \mathrm{~cm}^{-1}$, которая относится к поперечному (ТО) фонону. Такой вид спектров является характерным для слоев $\mathrm{GaSb}$ с ориентацией (001) [8].

Из спектров КРС видно, что положения линий LO-фонона для исходной подложки и для слоя с соотно- шением потоков $\mathrm{TMSb} / \mathrm{TEGa}=50$ совпадают с положением этой линии в объемном $\mathrm{GaSb}$ (пунктирная линия на рис. 5), что указывает на отсутствие деформации в них. В то же время для слоев с соотношением потоков $\mathrm{TMSb} / \mathrm{TEGa}=2$ и 10 наблюдается небольшой высокочастотный сдвиг $\left(\Delta \omega_{\mathrm{LO}}=0.6 \mathrm{~cm}^{-1}\right)$. При этом сдвиг LO-фононной линии не сопровождается существенным уширением, что позволяет сделать заключение, что данный эффект связан не с изменением концентрации свободных носителей. Можно предположить, что такой сдвиг может быть вызван наличием в образцах деформаций сжатия в плоскости слоев [8 и ссылки в ней]. Используя выражение из работы [8] $\Delta \omega_{\mathrm{LO}}=-3.7 \cdot 10^{2} \varepsilon_{\|}$, из величины сдвига можно вычислить значение деформации в плоскости слоя. Для $\Delta \omega_{\mathrm{LO}}=0.6 \mathrm{~cm}^{-1}$ значение деформации составляет $\varepsilon_{\|}=-1.6 \cdot 10^{-3}$.

Анализ спектров показывает, что для исходной подложки величина FWHM LO-фононной линии равна $3.9 \mathrm{~cm}^{-1}$. Для слоев $p$-GaSb, выращенных при соотношении потоков TMSb/TEGa $=2$ и 10, значения FWHM равны $3.5 \mathrm{~cm}^{-1}$, что может быть связано с улучшением кристаллической структуры эпитаксиальных слоев по сравнению с подложкой. Для слоя $p$ - GaSb, выращенного при соотношения потоков $\mathrm{TMSb} / \mathrm{TEGa}=50$, значение FWHM равно $3.7 \mathrm{~cm}^{-1}$. Это значение больше, чем для слоев с TMSb/TEGa $=2$ и 10, но меньше, чем для исходной подложки. Отсюда следует, что структурное качество слоев с $\mathrm{TMSb} / \mathrm{TEGa}=50$ ниже, чем для слоев c $\mathrm{TMSb} / \mathrm{TEGa}=2$ и 10, но выше, чем для исходной подложки. Это может быть связано с образованием в слоях $p$-GaSb c TMSb/TEGa $=50$ структурных дефектов. Таким образом, данные спектроскопии КРС подтверждают результаты рентгенодифракционных измерений.

\section{3. Заключение}

Разработана высокотехнологичная методика изготовления методом ГФЭМОС эпитаксиальных слоев $\mathrm{GaSb}$ (в том числе и высокоомных) в широком диапазоне соотношения потоков TMSb/TEGa. В слоях $p$-GaSb, выращенных при соотношениях потоков $\mathrm{TMSb} / \mathrm{TEGa}=2$ и 10, по данным спектроскопии КРС, рентгеноструктурных и ФЛ исследований, наблюдалось улучшение структурного качества по сравнению с подложкой $n$-GaSb:Te. При соотношении TMSb/TEGa $=50$ были выращены преднамеренно не легированные эпитаксиальные слои $\mathrm{GaSb}$, обладающие удельным электрическим сопротивление $>400$ Ом · см. Кристаллическое совершенство материала этих слоев, по оценкам различных исследований, несколько ниже, чем для лучших образцов (полученных при $\mathrm{TMSb} / \mathrm{TEGa}=2$ и 10 ), но соизмеримо с качеством используемых подложек. Проведенные исследования показали, что такие слои могут быть использованы для интегральных структур оптоэлектроники в качестве подложек с большим удельным сопротивлением. 


\section{Благодарности}

В заключение авторы выражают глубокую благодарность коллегам из ФТИ им. А.Ф. Иоффе, С.И. Трошкову за снимки сколов и поверхностей образцов, сделанные при помощи сканирующего электронного микроскопа (СЭМ) и В.Ю. Давыдову за поддержку и внимание к данной работе.

\section{Конфликт интересов}

Авторы заявляют об отсутствии конфликта интересов.

\section{Список литературы}

[1] P.S. Dutta, H.L. Bhat, V. Kumar. J. Appl. Phys., 81, 5821 (1997).

[2] А.Н. Баранов, Т.И. Воронина, Т.С. Лагунова, И.Н. Тимченко, 3.И. Чугуева, В.В. Шерстнев, Ю.П. Яковлев. ФТП, 23 (5), 780 (1989).

[3] P.S. Dutta, V. Prasad, H.L. Bhat. J. Appl. Phys., 80 (5), 2847 (1996).

[4] A. Bignazzi, E. Grilli, M. Guzzi, M. Radice, A. Bosacchi, S. Franchi, R. Magnanini. J. Cryst. Growth, 169, 450 (1996).

[5] P.S. Dutta, A.G. Ostrogorsky. J. Cryst. Growth, 197, 749 (1999).

[6] A.S. Vlasov, E.P. Rakova, V.P. Khvostikov, S.V. Sorokina, V.S. Kalinovsky, M.Z. Shvarts, V.M. Andreev. Sol. Energy Mater. Sol. Cells, 94, 1113 (2010).

[7] M. Hakala, M.J. Puska, R.M. Nieminen. J. Appl. Phys., 91, 4988 (2002).

[8] C. Raisin, A. Rocher, G. Landa, R. Carles, L. Lassabatere. Appl. Surf. Sci., 50, 434 (1991).

Редактор Г.А. Оганесян

\section{Growth gallium antimonide high-resistance by MOCVD method}

\section{R.V. Levin, A.S. Vlasov, A.N. Smirnov, B.V. Pushnyi}

loffe Institute,

194021 St. Petersburg, Russia

Abstract The results of studies of $p$-GaSb epitaxial layers grown by gas-phase epitaxy from metal-organic compounds with a TMSb/TEGa ratio from 1 to 50 are presented. At a ratio of $\mathrm{TMSb} / \mathrm{TEGa}=50, \mathrm{GaSb}$ epitaxial layers with a specific electrical resistance of $400 \Omega \cdot \mathrm{cm}$ were obtained. It is shown that the crystalline quality of such layers, which was evaluated by several methods, remained comparable to the quality of the substrates $(n-\mathrm{GaSb})$ used for $p$-GaSb growth. 